\title{
Self-Administration of Psychoactive Substances by the Monkey***
}

\author{
A Measure of Psychological Dependence \\ Gerald Denead***, Tomoji Yanagita ${ }^{+}$, and M. H. Seevers \\ Department of Pharmacology, The University of Michigan Medical School \\ Ann Arbor, Michigan
}

Received December 26, 1968

Final Version: June 16, 1969

Summary. A method has been developed which permits monkeys to self-administer drug solutions, at will, through indwelling intravenous catheters. Psychological dependence on the effects of a drug occurs when a naive monkey voluntarily initiates and maintains self-administration of the drug. If, in addition to psychological dependence, the drug also produces psychotoxicity, either directly or upon abrupt withdrawal, it has a potential abuse liability.

In the present study monkeys developed psychological dependence on morphine, codeine, cocaine, d-amphetamine, pentobarbital, ethanol, and caffeine. All of these drugs except caffeine produced psychotoxicity. Monkeys did not develop psychological dependence on nalorphine, morphine-nalorphine mixtures, chlorpromazine, mescaline or physiological saline.

Key-Words: Psychological Dependence - Psychotoxicity - Drug Abuse Drug Dependence - Psychoactive Drugs.

\section{Introduction}

In this laboratory an extensive experience concerning the behavioral effects of morphine-like drugs in the monkey (Macaca mulatta) has been obtained during the last 20 years. A continuing program on the evaluation of the physiological dependence capacity of morphine-like drugs is in progress, and over 700 compounds have been examined during this period. The biological response of the monkey to morphine-like substances, in most respects, parallels closely their actions in man, and

* Preliminary reports of this study were presented at the Fall Meeting of the American Society for Pharmacology and Experimental Therapeutics, 1964. Pharmacologist 6, 182 (1964).

** Supported by USPHS Grants MH 2814 and MH 5320 and by Committee on Problems of Drug Dependence, National Research Council, National Academy of Sciences.

*** Present Address: Southern Research Institute, Birmingham, Alabama 35205.

+ Present Address: Central Institute for Experimental Animals, 1433, Nogawa, Kawasaki, Japan. 
predictions for man, based upon monkey data, have been proved to be qualitatively accurate. Although physiological dependence is a very important negative reinforcing factor in inducing drug-seeking behavior to avoid the aversive effects of withdrawal, it develops only to substances which have a predominantly depressant action on the central nervous system, e.g., morphine and its derivatives, barbiturates and the pharmacologically related substances, alcohol and other hydrocarbons. Yet, many stimulants such as cocaine and amphetamine do not induce physiological dependence but are highly reinforcing in man and have equal, if not greater, capacity for abuse leading to psychotoxicity. Unfortunately, no animal model currently exists for the evaluation of what is generally termed psychological dependence (i.e., the voluntary initiation and maintenance of self-administration of drugs).

Investigations involving self-administration of drugs by animals are designed usually to determine whether the animal will drink water or a drug solution. This technique has had extensive applications in small animals, mainly mice and rats, with alcohol, morphine and other substances. Beach (1957), Nichols et al. (1956) and Wikler et al. (1963) have all demonstrated that rats will show a preference for a solution of morphine or other opiate over water, or for conditions formerly associated with the experience of morphine's effects, but only after they have been rendered physiologically dependent to this drug by injection. In 1960, studies were initiated in this laboratory involving self-administration of morphine-like substances by the monkey in drinking water. These experiments demonstrated that some monkeys, but not all, would choose morphine solutions over water and continue self-administration until physiological dependence had developed. Other morphine derivatives like methadone, meperidine and levorphanol were not acceptable, even to morphine-dependent animals, thus imposing serious limitations on the general applicability of the oral method in the monkey.

In 1962, Weeks described a technique for intravenous self-administration of drugs in the rat. He demonstrated clearly that rats, rendered physiologically dependent by programmed intravenous administration of morphine, will then maintain the dependent state by pressing a lever to obtain the drug. Since this technique appeared to offer a great potential for studies in the monkey, with possible long-range application as a screening method for psychological dependence, studies were initiated to develop a similar technique for this species. The present paper describes this technique and presents a preliminary survey of representatives of the principal drugs which are strongly reinforcing for man and are subject to widespread abuse. In 1964, Thompson and Schuster administered morphine intravenously to monkeys restrained in an experimental chair and were able to induce self-administration patterns for morphine 
by lever-press. Their experiments were designed primarily to compare and evaluate the reinforcing capabilities of morphine versus food, and did not attempt to determine whether the monkey would spontaneously initiate self-administration following the first drug experience.

The present experiments were designed, therefore, to determine whether a monkey, having received the first injection of any drug by spontaneous lever-press, would continue to seek reinforcement by increasing the number of lever presses and maintain such a pattern of selfadministration for long periods of time. If the monkey fulfilled these requirements, we assumed that psychological dependence had been demonstrated.

\section{Apparatus}

Housing and Restraining. The monkey is housed in an open-front cubicle. Each cubicle is 36 inches high, 30 inches wide, and 26 inches deep. These are constructed in double-deck units of four cubicles each. The side walls and tops of these cubicles are made of 5/16-inch opaque tempered polyethylene to permit maximum light. The floor is made of 1-inch mesh screen, with a drop-pan below, which is flushed automatically every 4 hours for sanitation and odor control.

A panel which is mounted in the center of the back of the wall contains two switches which the monkey can press to activate the injector. Pressing of one of these switches results in an intravenous injection of a drug solution to the monkey. Pressing the other causes the solution to be delivered back into the drug reservoir while all sounds and other external clues are identical to those accompanying an actual injection. The wiring to the two switches can be interchanged at will by the experimenter. A drinking fountain for tap water is also mounted on the back wall of the cage. A specially-prepared Purina monkey chow containing $183 \mathrm{gm} /$ ton isoniazid, as a tuberculosis preventitative ${ }^{1}$, is supplied from a box beneath the perch in a rear corner of the cubicle.

The monkey is restrained in this cubicle by a specially-designed metal harness (Fig.1) and restraining arm (Fig.2) which affords maximum movement in order to permit exercise and to eliminate to some extent the physical effects of restraint. The restraining arm, attached in the left rear corner of the cubicle, is made of stainless steel tubing and is patterned after the human arm. A permanent silastic catheter passes through the arm from the injector to the harness housing where it is attached to the catheter implanted in the animal. The "shoulder" (joint A) permits swivel and hinge action of the whole arm; the "elbow" (joint B), hinge and rotation of the forearm of about 300 degrees; the "wrist" (joint $\mathrm{C}$ ),

1 To provide an average daily dose of $20 \mathrm{mg} / \mathrm{kg}$ isoniazid; $6.8 \mathrm{gm} /$ ton pyridoxine $\mathrm{HCl}$ is also added to prevent a deficiency state of the latter. 


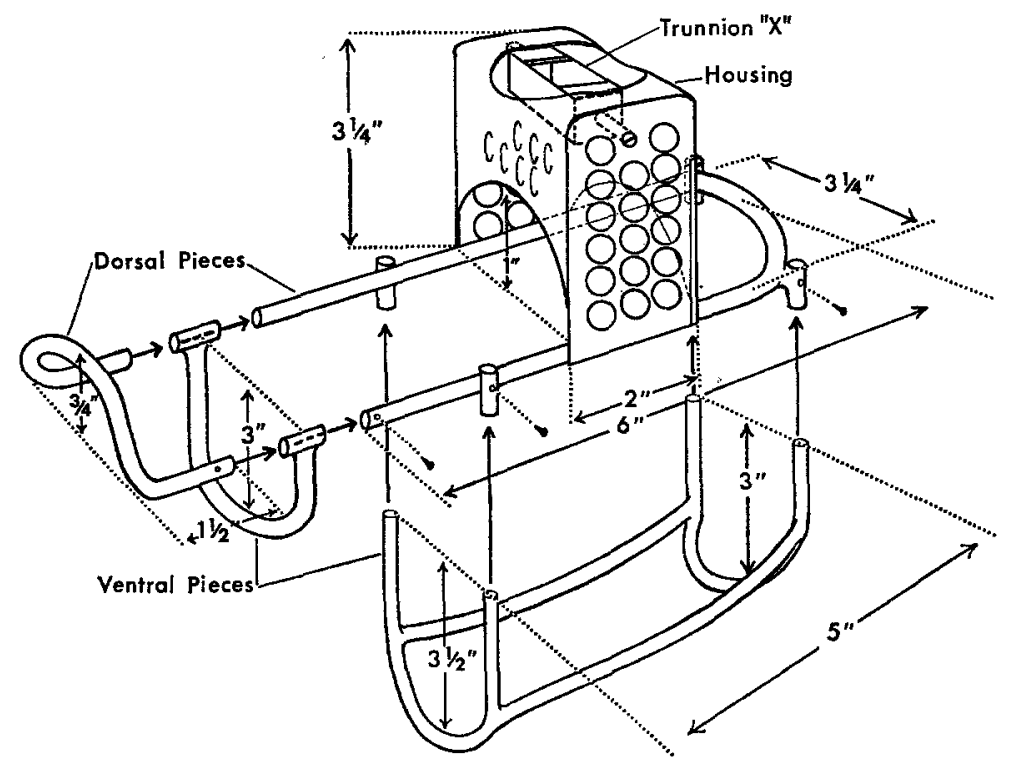

Fig.1. Exploded perspective view of the harness designed to fit a medium-sized $(4.0-4.5 \mathrm{~kg})$ rhesus monkey. The harness is constructed of $0.067 \mathrm{in}$. stainless steel tubing

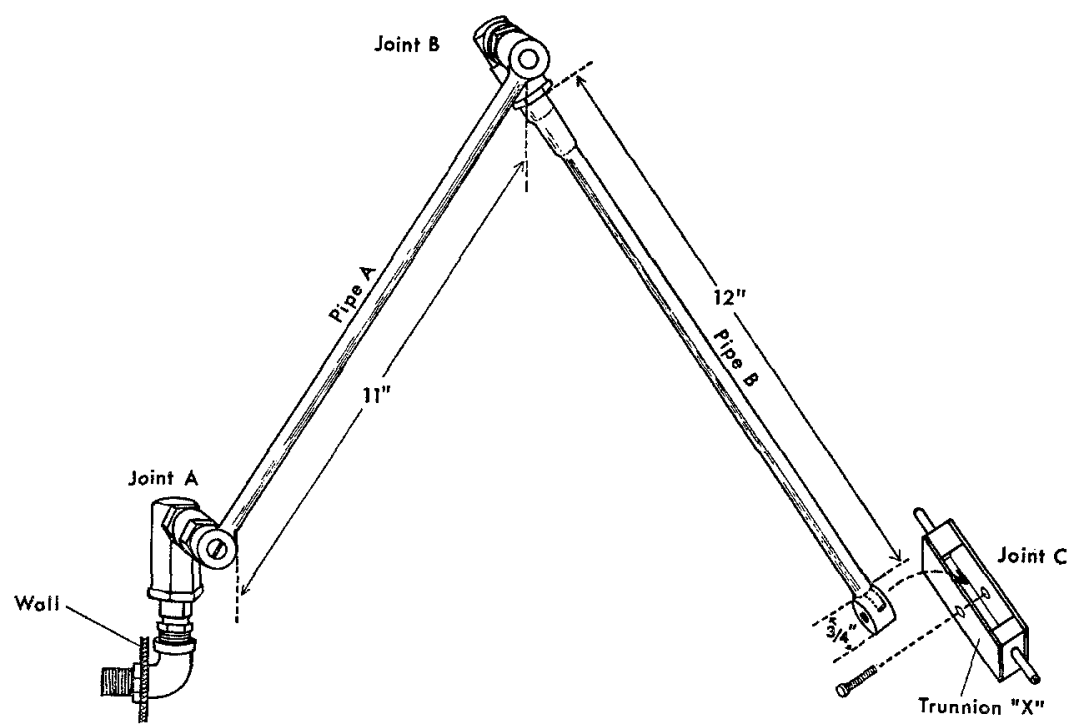

Fig.2. Perspective view of the restraining arm employed to connect the monkey's harness to the cage wall. The joints, $A$ and $B$, are modified swivels from pressurized lubricating lines. These are available from any automative supplier

3 Psychopharmacologia (Berl.), Bd. 16 


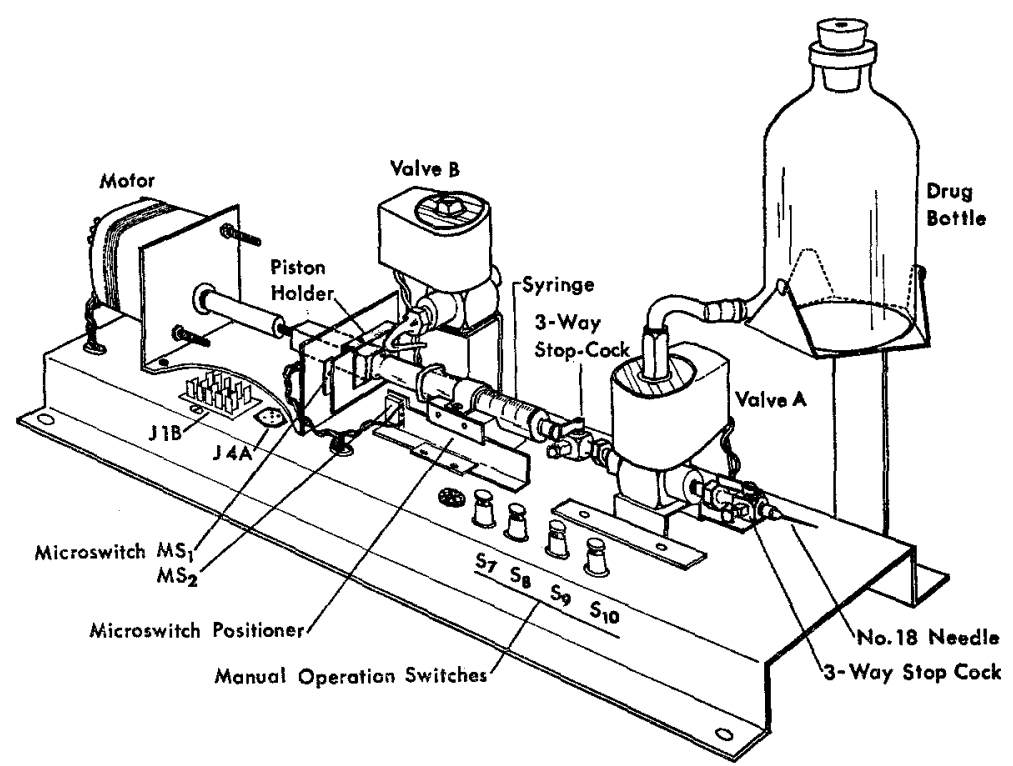

Fig. 3. Perspective view of the injector. Microswitches $\left(\mathrm{MS}_{1}\right.$ and $\left.\mathrm{MS}_{2}\right)$ are limit switches which serve to stop and to reverse the motor, respectively. Valve $A$ is a 3-way solenoid valve which permits the syringe to refill automatically from the reservoir bottle. Valve $B$ is a 2 -way solenoid valve which delivers a small stream of water, as a lubricant, to the syringe when the injector is activated

both horizontal and vertical hinge action with the harness. The harness, also made of stainless steel tubing, is adjustable within limits, although several sizes are necessary in order to accommodate different sized animals.

Drug Infusion Unit. The injector is illustrated in Fig. 3. It may be activated by the monkey pressing the lever in the cage or by predetermined programming. Activation of the motor-driven injector drives the solution from the syringe into the catheter which traverses the restraining arm to the back of the monkey, passes subcutaneously, enters the jugular vein and terminates near the right atrium of the heart. The position of the catheter tip near the right heart offers the most favorable site since the large blood volume and continued turbulence assures rapid dilution and minimal clot formation.

One ec fluid is injected within $25 \mathrm{sec}$ and another $25 \mathrm{sec}$ is required to refill the syringe from the drug reservoir bottle before the next injection. is available. This delay imposes a limit in the frequency of injection which may be a factor in establishing accurate reinforcement rates with low drug concentrations. 
Programming and Recording. The injector may be controlled in four ways: 1 . by the monkey alone; 2 . by automatic timer alone; 3 . by the timer if the monkey fails to press the lever within a predetermined period; 4 . by the monkey, with a timer blocking the circuit after injection, preventing the monkey from taking the next injection until a predetermined time has elapsed. Other programs, such as a fixed or progressive ratio of lever presses for injections, require external accessory units to be attached to the main control unit. The number and time of day of each injection, self-administered or programmed, and the times of lever depression, whether reinforced or not, are recorded on an event recorder.

Presurgical Care. A conditioned monkey weighing from $3^{1 / 2}$ to $4 \mathrm{~kg}$ is chosen from the colony and a harness is fitted. The monkey usually adapts itself to the restraining device within a day or two. Highly excitable monkeys, when restrained for the first time, may make violent efforts to escape, and in some instances have broken the restraining arm. To avoid this, a heavy-duty training arm is used and the front of the cage covered with plexiglas to prevent escape. If the harness does not fit well, or is improperly adjusted, sores will develop around the shoulders or on the back of the monkey. A loose or poor fit also allows the monkey to insert his foot under the harness and disconnect or dislodge the catheter. Proper fitting can be accomplished within a week.

Catheter Implantation. A silicone rubber catheter, $2.2 \mathrm{~mm}$ OD and $1.0 \mathrm{~mm}$ ID, is inserted aseptically into the jugular vein after exposure by neck incision under barbitutate anesthesia. Proper location of the tip of the catheter near the right atrium of the heart is aided by either of two methods: 1 . recording the blood pressure at the tip of the catheter with a strain-gauge manometer, or 2. recording the EKG from the external tip of the saline-filled catheter. Venous pressure in the superior vena cava is approximately zero or a few millimeters negative. When the catheter enters the atrium, pulse pressure is discernable to the order of zero diastolic to a few $\mathrm{mm}$ systolic. In the right ventricle, the pulse pressure is greater. The $P$-wave of the EKG serves as a useful indicator of the position of the tip of the catheter. $\Delta s$ the catheter arrives at the heart and enters the atrium, the $\mathrm{P}$-wave is negative but becomes positive as it nears the $A V$ valve. If the tip enters the ventricle, the $Q R S$ complex shows a dramatic increase in voltage. When it is ascertained that the catheter is in the atrium, it is withdrawn $1 \mathrm{~cm}$ and tied in place.

After the catheter is properly placed, it is also fixed at its entrance point into the jugular vein by ligatures cemented to the catheter $10 \mathrm{~cm}$ from the tip in advance of surgery. The free end is then drawn subcutaneously by a probe stylet to the intended point of exit through the skin. 
of the back where it is attached to the catheter which traverses the restraining arm.

Post-Surgical Care. When surgery is completed, the monkey is then returned to the cage and programmed injections of saline solutions are begun at intervals of $\mathbf{3}$ to $\mathbf{6}$ hours until his surgical wound heals, usually within 5 days. The animal is then ready for experimentation.

Experimental Procedures. Upon surgical recovery, two lever switches are installed in the rear panel, one of which is arranged to deliver saline when activated by the monkey. If the monkey presses the other lever this also activates the injector but the saline (or drug solution) is delivered back into the reservoir rather than into the monkey. The switch connections on the two levers are easily interchangeable. A signal light is also installed above the levers. The light, when on, indicates to the monkey that he can activate the injector and receive an injection by pressing the lever switch. For the first few days during injections of saline, a clear glass lens is placed over the light. During this period the monkey, a naturally curious animal, will depress a lever. When the baseline rate of lever pressing has been observed, the saline solution is replaced by drug solution and a colored lens is placed over the panel light. The next depression of the proper lever by the monkey results in the injection of a test drug. If the monkey is lever-pressing for saline even at a low rate at the end of the control period, it is only necessary to substitute drug solution for saline in order to give the monkey his first drug experience. If the lever-pressing for saline has become extinguished, it is necessary to use another device to initiate lever pressing when the drug is substituted. This is done by taping a raisin or small piece of candy to the lever with transparent cellulose tape. In his efforts to obtain the raisin, the monkey will activate the injector. If the test drug is positively rewarding, two or three such trials should teach the monkey that lever-pressing is associated with drug effects and he will increase the number of lever presses until a pattern of drug self-administration is established. If the drug is aversive, or not rewarding, the monkey will not attempt to extricate raisins or candy from beneath the tape in subsequent trials even though he will take the raisins from the investigator's hand.

When a monkey fails to initiate self-administration of a test drug, it becomes pertinent to know if psychological dependence might develop after further exposure to the drug's effect. To test this possibility, injections are programmed automatically at constant intervals, while at the same time the injector can also be activated by the monkey. If the monkey's initial indifference to the drug changes to a positive preference, this change is indicated by monkey-activated injections during intervals between programmed injections. If self-administration does not 
begin within a month, the monkey is assumed not to have developed psychological dependence to this particular drug.

The concentration of drug solution in water or in $0.9 \%$ saline is prepared in such a manner that the unit dose per kilogram administered. at each injection is contained in $0.25 \mathrm{cc}$ of solution, except for alcohol which is prepared in a $20 \%$ solution by weight.

Dosages. All dosages are expressed in terms of the common salts of the drugs employed.

\section{Results}

Morphine. Morphine was the first drug studied using this technique. Eleven naive animals were studied, three being retested at two dosage levels. Four monkeys failed to initiate lever- pressing at a dose of $0.5 \mathrm{mg} /$ $\mathrm{kg}$ and three out of ten failed at $2.5 \mathrm{mg} / \mathrm{kg}$. When programmed injections at 4-hour intervals were begun, these latter three soon initiated selfadministration and ultimately achieved as high a daily intake as the other seven.

Fig.4 shows the self-administration pattern of a typical animal during a 27-week period. All animals increased their daily intake steadily through the 6th or 7th week and then maintained a fairly stable daily dosage between 50 and $100 \mathrm{mg} / \mathrm{kg}$. One exceptional monkey continually increased his dose for 30 weeks and finally attained an intake of $280 \mathrm{mg} / \mathrm{kg} /$ day. Although some individual variation existed, the pattern of self-administration of morphine was characterized by large diurnal and small nocturnal intake. Some monkeys slept through the entire period of 12 to 8 a.m., when the laboratory lights were extinguished, without self-administering any morphine.

In no instance did any animal voluntarily discontinue self-administration of morphine. In a few instances, when injections were interrupted by catheter kinking, plugging or breaking, or by mechanical failure, the monkeys showed typical and severe abstinence syndromes during which they pressed the lever almost continuously in attempts to obtain morphine injections.

During the period of escalating dosage the animals were drowsy and apathetic but as the dosage stabilized these animals, though less active than controls, appeared normal to casual observation. As with all morphine-dependent animals, food intake was reduced initially and some temporary weight loss occurred. The longest experiment in this series was continued for a period of 16 months. Barring infections these animals maintained good health. Bacterial endocarditis with pulmonary emboli was the commonest complication.

Codeine. Four of five monkeys initiated lever-pressing for codeine at doses of 0.5 and $2.5 \mathrm{mg} / \mathrm{kg}$ and gradually increased the rate of intake 

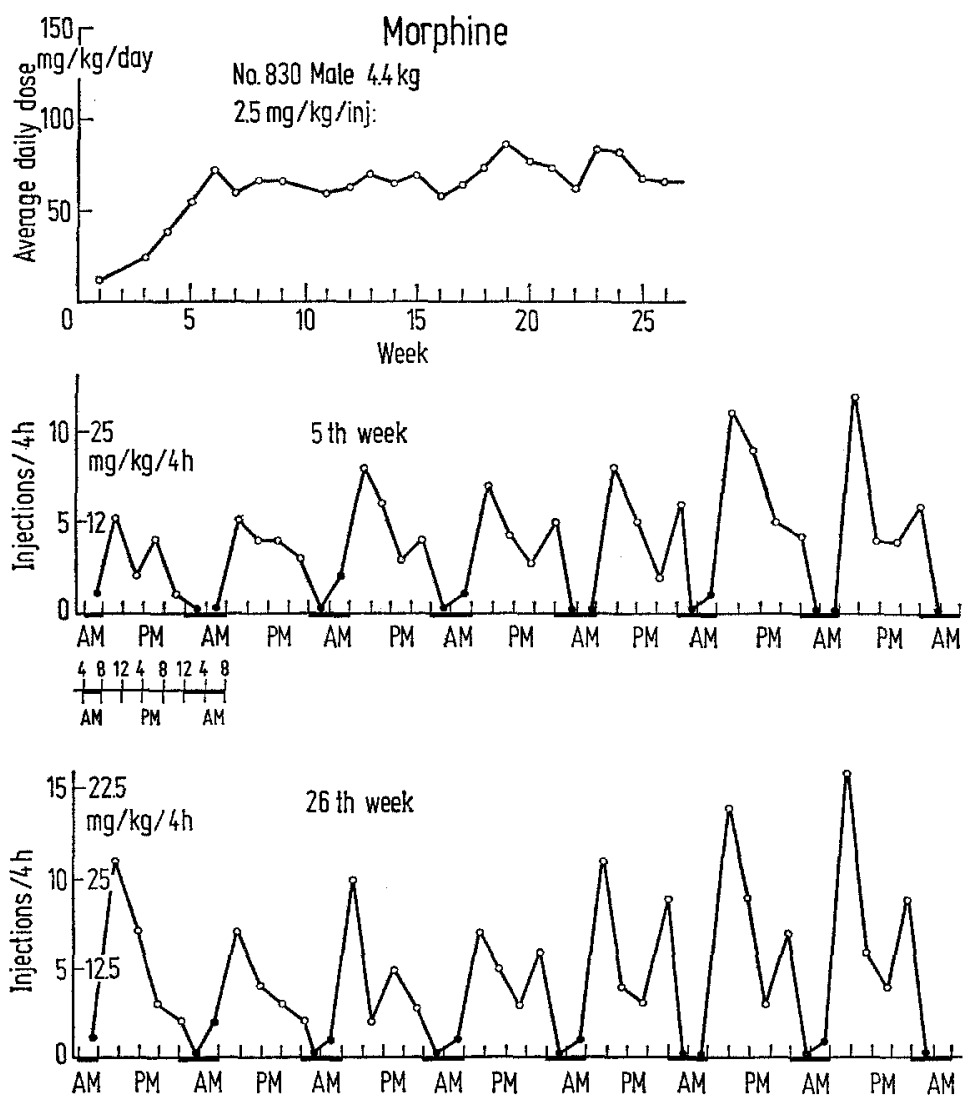

Fig.4. Typical morphine self-administration pattern. The upper graph illustrates the fairly constant dosage level attained after a 6 -week period of rapid incrementation. The lower graphs illustrate the day-to-day consistency of morphine selfadministration

until a high plateau was reached in 5 to 6 weeks (see Fig.5). The fifth animal required automatically-timed "priming" injections before selfadministration behavior developed.

Codeine, administered intravenously, produced slight depression initially but as the dosage level increased the monkeys became increasing excitable and alert. The pattern of self-administration differed from that of morphine in that no significant nocturnal remission was observed. Voluntary abstinence was never observed. Mechanical failure on the 18th day of self-administraton, at a daily dosage level of $80 \mathrm{mg} / \mathrm{kg}$, resulted in the appearance of a mild morphine-like abstinence syndrome. The highest average daily dosage observed for one week was $600 \mathrm{mg} / \mathrm{kg}$. 


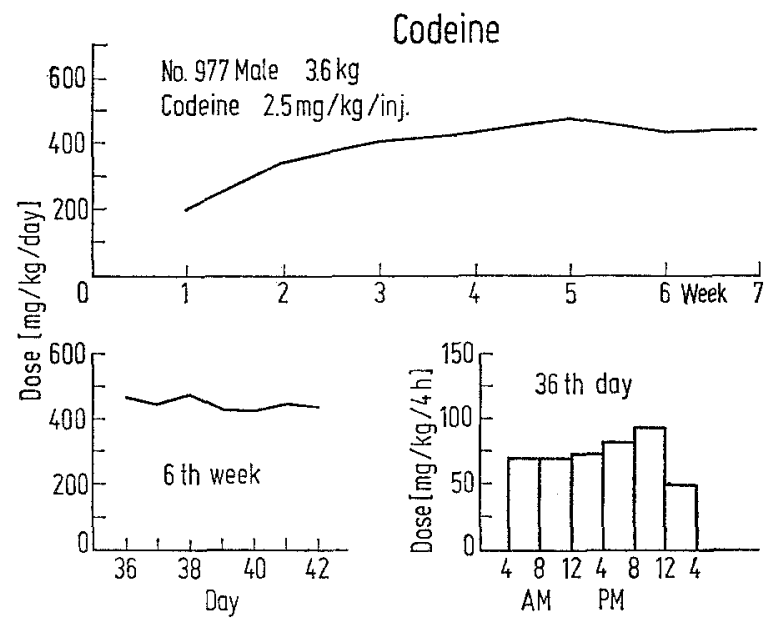

Fig. 5. Typical codeine self-administration pattern. The graphs illustrate the weekto-week, day-to-day and period-to-period consistency of codeine self-administration

This monkey died of convulsions during the succeeding week at a dosage level of $670 \mathrm{mg} / \mathrm{kg}$ on that particular day. All five monkeys died within 6 to 8 weeks of unrestricted intake of codeine. Three were observed to die in convulsions due to overdosage, not abstinence. The others were found dead in the morning and were presumed to have died in convulsions since they appeared to be in good health on the previous day and showed no specific cause of death at autopsy.

Nalorphine. Self-administration of nalorphine did not occur in any of four monkeys at a dose of $2.5 \mathrm{mg} / \mathrm{kg}$. After the first drug experience each monkey refused to press the lever even with raisin inducement. Timer-programmed injections of $2.5 \mathrm{mg} / \mathrm{kg}$ every 4 hours were then administered for 1 month. No monkey initiated self-administration during this period nor upon cessation of the programmed administration. During nalorphine administration the monkeys were less active, somewhat apprehensive and salivated mildly for $10-15 \mathrm{~min}$ after each injection. Upon withdrawal, excessive yawning and scratching were observed for 2 days.

Morphine-Nalorphine Mixture. A mixture of $2.5 \mathrm{mg} / \mathrm{kg}$ each of morphine and nalorphine was tested in 4 monkeys. These monkeys had all previously demonstrated psychological dependence to morphine alone but had not been permitted to self-administer morphine to the point of developing physiological dependence. None initiated voluntary selfadministration of the morphine-nalorphine mixture.

Cocaine. Two of four monkeys initiated self-administration of $0.25 \mathrm{mg} / \mathrm{kg}$ cocaine per dose. Three others (two of which had not initiated 

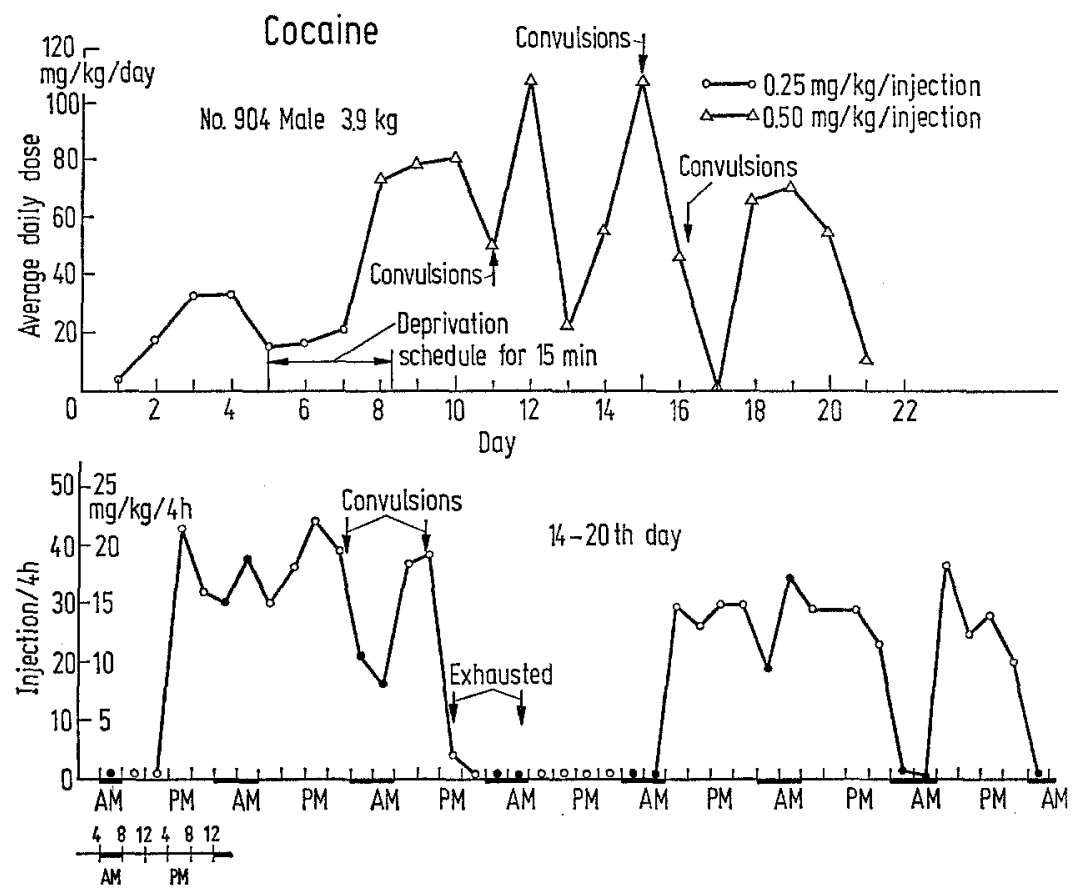

Fig.6. Typical cocaine self-administration pattern. The graphs illustrate the erratic course of cocaine self-administration

at $0.25 \mathrm{mg} / \mathrm{kg}$ ) initiated and maintained self-administration at a dose of $1.0 \mathrm{mg} / \mathrm{kg}$. Once cocaine self-administration was initiated, the course of intake was of rapid but erratic increase culminating in convulsions and death within 30 days. In order to prolong the experiments beyond 30 days it was necessary to restrict the intake to 1 dose per hour. Fig. 6 illustrates a typical example in which the intake was unrestricted. The dosage pattern was characteristic; cocaine was self-administered around the clock until exhaustion occurred ( 2 to 5 days in these experiments). The maximum intake during any 24-hour period was $180 \mathrm{mg} / \mathrm{kg}$. Upon exhaustion, self-administration was voluntarily discontinued for periods ranging from 12 hours to 5 days during which the monkeys slept fitfully and ate frequently. Cycles of self-administration were then resumed.

During self-administration of cocaine the monkeys became apprehensive and restless, showed almost constant choreiform movements, stereotypy, dysmetria, tremors, mydriasis, pilo-erection and gross ataxia during the first several days. As the dosage increased in daily amount and duration, signs of somatic and psychotoxicity increased. The monkeys showed an extremely rapid loss of muscle mass and grand mal con- 
vulsions became frequent. Behavior consistent with visual hallucinations (staring and grasping at the wall) and tactile hallucinations (continued scratching and biting of the skin of the extremities, to the point of producing extensive wounds and even amputation of the digits) was consistently observed. The monkeys also appeared to become unaware of their surroundings in that they ignored raisins and candy which were proffered by the experimenters. These manifestations of toxicity were rapidly reversible when cocaine administration was discontinued. During periods of high drug intake all monkeys pressed the inactive lever as frequently as the active lever.

Morphine-Cocaine. Four monkeys were implanted with dual-lumened catheters. The lumens were connected to two separate injectors which in turn were activated by separate lever switches in the monkeys' cages. One injector supplied $2.5 \mathrm{mg} / \mathrm{kg}$ morphine per dose, and the other supplied $1.0 \mathrm{mg} / \mathrm{kg}$ cocaine. The monkeys could self-administer either or both drugs as they wished by pressing the appropriate lever.

Within a few days all four monkeys established a pattern of selfadministration of both drugs, cocaine being the predominant choice during the day and morphine during the late evening and night. Within 7-10 days the monkeys became so disoriented that no particular dosage pattern was discernable. Although fewer convulsions developed than when cocaine was the only drug of self-administration, combined toxic effects of the two drugs consisted of delirium, marked motor impairment, anorexia and emaciation. The experiments terminated in death within $2-4$ weeks.

The Amphetamines. Five monkeys initiated and maintained selfadministration of $0.1 \mathrm{mg} / \mathrm{kg}$ d-amphetamine. The frequency of selfadministration was rapidly increased until a maximum daily dosage of $9 \mathrm{mg} / \mathrm{kg}$ was attained in $2-3$ weeks. Self-administration of amphetamine was characterized by irregularity of daily intake. Periodic voluntary withdrawals lasted from 1 day to 2 weeks. During periods of high intake self-administration continued day and night. Substitution of methamphetamine $(0.1 \mathrm{mg} / \mathrm{kg} /$ dose $)$ in two of the monkeys for 5 weeks did not produce any detectable change in the behavioral pattern.

The somatic and behavioral toxic effects were similar to, but somewhat milder than, those of cocaine. No grand mal convulsions were observed nor did chewing of the forearms or digits occur. One monkey did pluck all of the hair off his arms and abdomen, however, indicating that mild tactile hallucinations may have been present. As with cocaine, all monkeys became confused and pressed both levers indiscriminately during periods of high dosage. The catabolic effect associated with cocaine administration was also observed in the monkeys which self-administered the amphetamines. 

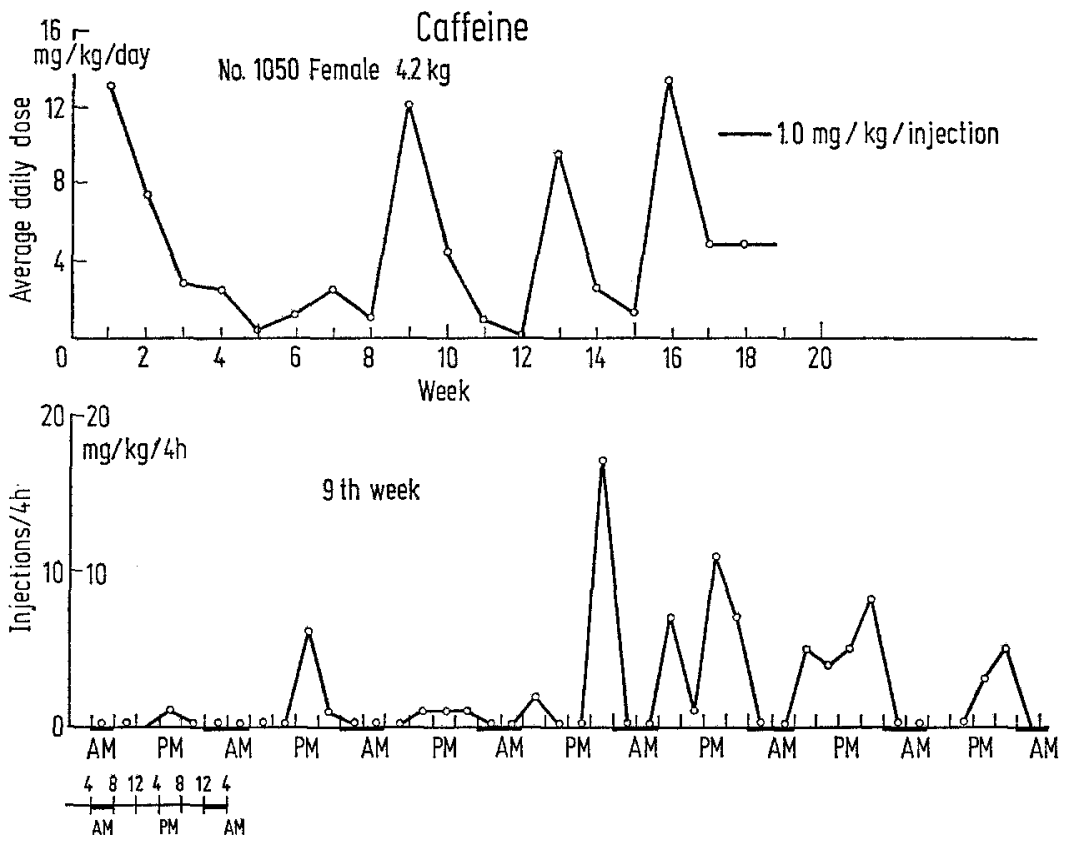

Fig. 7. Typical pattern of caffeine self-administration illustrating the unpredictable course of drug intake

Caffeine. Two monkeys failed to initiate self-administration of $1.0 \mathrm{mg} /$ $\mathrm{kg}$ caffeine. One initiated and one failed to initiate self-administration at $2.5 \mathrm{mg} / \mathrm{kg}$ although "priming" with automatic injections caused the latter to begin self-administration. At a dose of $5.0 \mathrm{mg} / \mathrm{kg}$, one monkey of four initiated self-administration voluntarily and two others did so with priming.

The pattern of self-administration of caffeine was in all cases sporadic -irregular intervals of self-administration alternated with periods of voluntary abstinence. There was no tendency to increase the dose nor to self-administer the drug at night. None of the monkeys showed any overt signs of drug effect or of toxicity during periods of self-administration of caffeine nor upon withdrawal. A typical experiment is illustrated in Fig. 7.

Mescaline. None of four monkeys initiated self-administration of mescaline either spontaneously or after 1 month of programmed administration at doses of 1,5 or $10 \mathrm{mg} / \mathrm{kg}$ every 2 hours. Salivation was observed after each injection indicating nausea although none of the monkeys vomited. The animals showed mydriasis and pilo-erection and were very 

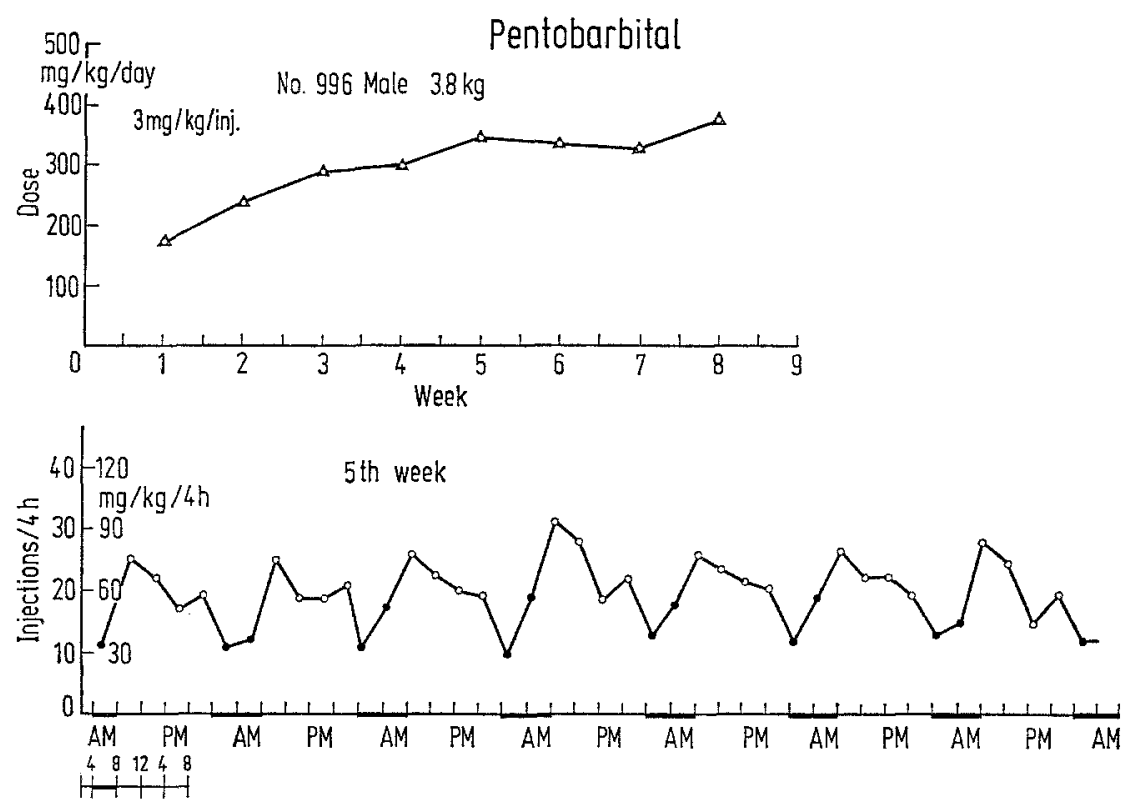

Fig. 8. Typical pattern of pentobarbital self-administration. The graphs illustrate the predictable and nearly maximal pattern of self-administration of pentobarbital

apprehensive, even of common laboratory noises. No abstinence signs were observed upon discontinuation of the programmed administration.

Pentobarbital. Five monkeys initiated and maintained self-administration of $3 \mathrm{mg} / \mathrm{kg}$ doses of pentobarbital. By the end of 1 week all monkeys were taking the maximum dose they were physically capable of selfadministering. As soon as they recoverved sufficiently from the last incapacitating dose, they immediately struggled to depress the lever switch. As time elapsed, tolerance developed, enabling the monkeys to increase their total daily intake until a plateau was reached in 5 or 6 weeks. The maximum average daily dose for any single week was $420 \mathrm{mg}$ / kg. A typical experiment is illustrated in Fig. 8. Self -administration was maintained during the night at approximately half of the daytime level of intake. All monkeys abstained 30-45 min each morning and each afternoon while they consumed greater than average meals. The animals maintained good physical condition and gained weight throughout the course of the experiment.

Prolonged voluntary abstinence never occurred. In the event of mechanical failure or deliberately conducted withdrawals, very severe abstinence syndromes were observed within $4-6$ hours. The syndromes 

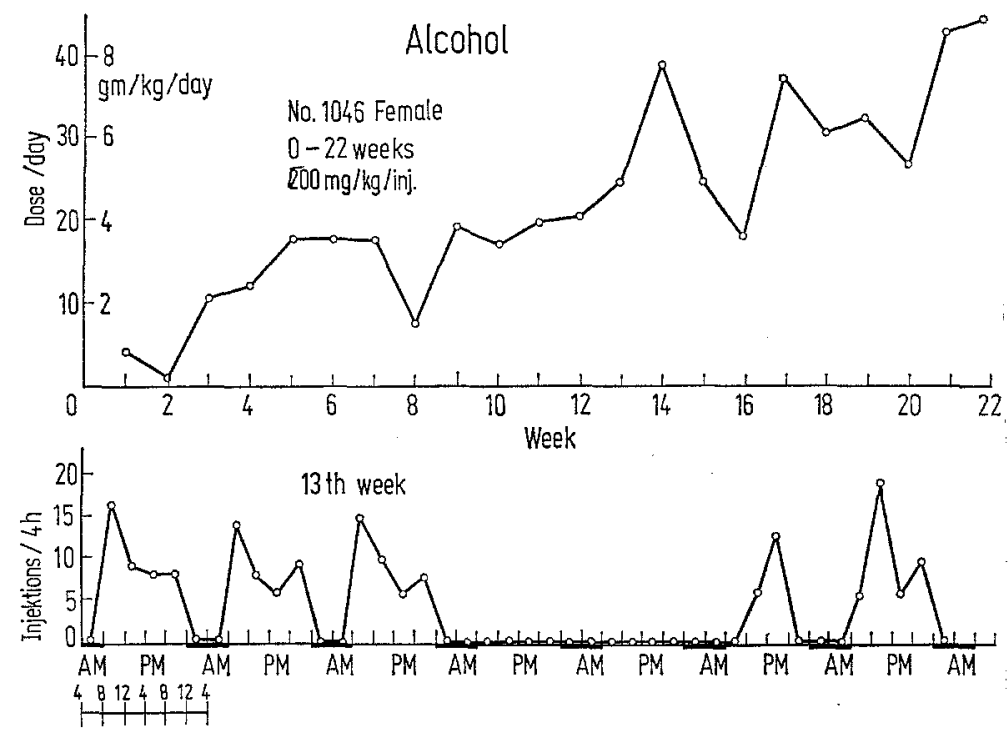

Fig. 9. Typical pattern of alcohol self-administration. The graphs illustrate the periodic voluntary abstinence interspersed between periods of high dosage

were characterized by extreme restlessness, tremors, grand mal convulsions and apparent hallucinations.

Ethanol. Four of five monkeys initiated self-administration of ethanol at a dose of $200 \mathrm{mg} / \mathrm{kg}$. The refractory monkey did not initiate selfadministration even after programmed injections of $200 \mathrm{mg} / \mathrm{kg} / \mathrm{hr}$ for 4 weeks. One which initiated self-administration discontinued after one month and never resumed. The maximum intake of those which maintained self-administration was $8.6 \mathrm{gm} / \mathrm{kg} /$ day for a 1 -week period.

In spite of the occurrence of severe abstinence syndromes, the monkeys voluntarily abstained for periods of 2 to 4 days through the first 4 months of self-administration; thereafter such periods of voluntary withdrawal seldom exceeded 24 hours. (See Fig.9).

During self-administration the monkeys showed severe motor incoordination and stupor, even to the point of light anesthesia. During withdrawal, a characteristic abstinence syndrome with tremor, vomiting, hallucinatory behavior and convulsions occurred as soon as $\mathbf{6}$ hours after the last dose.

Food intake during the course of ethanol self-administration was minimal; all monkeys showed marked weight loss and cachexia.

Two monkeys died during the course of the experiments due to suffocation from respiratory obstruction during anesthesia. 


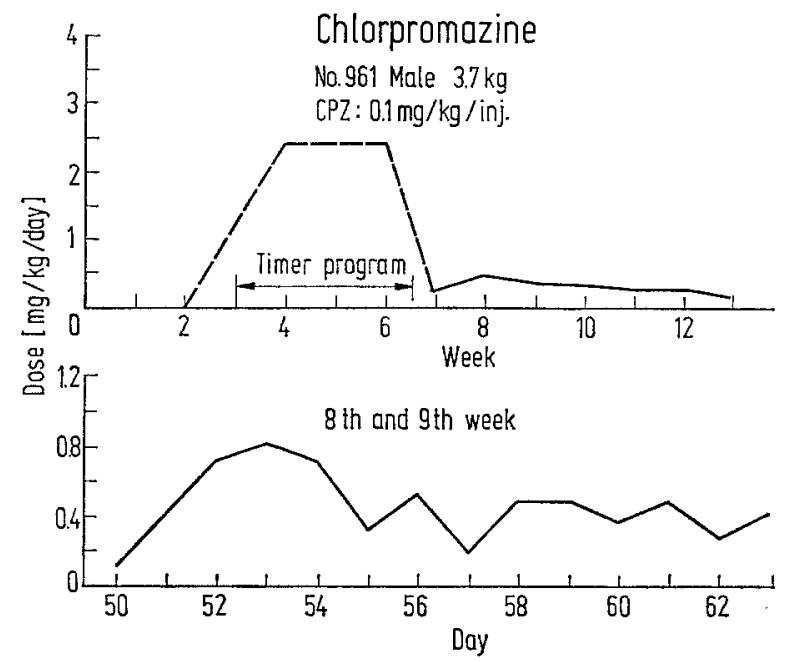

Fig.10. Pattern of chlorpromazine self-administration observed in 2 of 6 monkeys after periods of automatically programmed injections

Chlorpromazine. None of six monkeys initiated self-administration of chlorpromazine at doses of 0.1 or $0.5 \mathrm{mg} / \mathrm{kg}$. The three at $0.5 \mathrm{mg} / \mathrm{kg}$ received programmed injections every 2 hours for 4 weeks and even then failed to take any additional injections voluntarily. Upon withdrawal of programmed injections however, two of these monkeys took 2 to 5 injections per day for several weeks and then abstained completely (see Fig.10).

During programmed administration, the monkeys showed typical phenothiazine effects of reduced spontaneous activity and responsiveness, narrowed palpebral fissures and slight miosis but no major dyskinesias were observed. Upon withdrawal no signs which could be construed as an abstinence syndrome were observed.

Saline. Throughout the study frequent attempts, including the use of raisin inducements, were made to establish physiological saline as a reinforcing agent in naive monkeys; none was successful.

\section{Discussion}

Previous investigations which involved self-administration of drugs by laboratory animals have employed subjects already made physiologically dependent upon the drug under study - in most instances, morphine. Interpretation of subsequent self-administration of the drug by the subjects is complicated by the existence of the state of physiological dependence. It is possible that the animals might have been motivated 
by either of two distinct, but quite different, mechanisms-escape avoidance, in which the ameliorating effect of injected morphine upon the distress of the abstinence syndrome was quickly learned, or positive reinforcement, in which, under the circumstances prevailing, the animals preferred to exist under the influence of the drug's effects.

The initial conditions of the present experiments make it possible to distinguish which of the alternative motivations leads to the animals, self-administration of drugs. The use of naive, rather than physiologically dependent, animals rules out the escape-avoidance mechanism since no distressing abstinence syndrome existed at the beginning of the experiments when the self-administration of the various drugs began. Under such conditions a monkey's refusal to self-administer a drug or a monkey's development of self-administration behavior is, in either case, the monkey's unhindered decision and a direct reflection of his preference. Since a majority of monkeys initiated and maintained self-administration of morphine, codeine, pentobarbital and ethanol, one must conclude that they developed psychological dependence-a preference to exist under the influence of the drugs' effects-before sufficient time had elapsed or dosage administered for physiological dependence to develop. It is quite likely, however, that as physiological dependence developed, the relief of the distress of the abstinence syndrome, afforded by self-administration of the drug, soon became a secondary motivation for continuing the selfadministration behavior.

The conclusion that psychological dependence is the primary motivation for drug abuse is further strengthened by the fact that monkeys selfadministered cocaine and d-amphetamine, neither of which produces physiological dependence. With such drugs the only possible motivation for continued self-administration is psychological dependence. Seevers (1968) has discussed this point at length in a recent symposium.

In the present experiments the monkeys self-administered those drugs which man abuses severely, yet they developed minimal or no selfadministration behavior for nalorphine, chlorpromazine and mescalinedrugs which people seldom abuse. The manifestations of somatic and behavioral toxicity observed during drug administration, and in some cases during withdrawal periods, were similar to the well-known toxicities of these drugs in man. If such parallelisms should continue as similar studies are conducted with representative members of the remaining classes of psychoactive drugs, this technique could prove to be valuable for predicting potential abuse liability of new psychoactive agents. The major limitation of the present technique is that drugs which are not water soluble cannot be tested. The evaluation of such important substances as the active ingredients of marihuana must await further refinements of methodology. 
These experiments illustrate that even with such drugs as morphine and cocaine, marginal doses exist to which some monkeys will develop psychological dependence while others will not. Even with higher, and presumably more rewarding, doses it was necessary to administer frequent doses automatically for variable periods of time before some monkeys began to self-administer the drugs. This individual variability in susceptibility to drug abuse is analogous to known variability in man. Individual monkeys may be classified as "susceptible" or "indifferent" or "resistant" to drug abuse, according to the readiness with which they develop psychological dependence. This fact should be of importance in behavioral studies which attempt to associate personality characteristics with tendencies to abuse drugs. It is also noteworthy that, to some extent at least, dose-response relationships exist with this complex behavioral phenomenon.

Some caution should be expressed in interpreting the results of studies such as these. The fact that monkeys will self-administer a drug is insufficient evidence on which to assess the drug's abuse liability. Of more relevance to abuse liability is the toxicity, somatic and behavioral, which results from self-administration or withdrawal of the drug. Though all five of the monkeys which were employed in the caffeine test eventually self-administered the drug, none showed any somatic or behavioral toxicity either during self-administration or upon withdrawal. This is in marked contrast to the toxicity associated with self-administration of morphine, pentobarbital, ethanol, cocaine, amphetamine, etc. While a drug must be self-administered before it is abused, a total assessment of its potential danger cannot be made from the fact that psychological dependence, as manifested by some degree of self-administration, occurs.

Although this technique was developed for the express purpose of studying psychological dependence, there are many other potential applications for which it would be useful. These range from behavioral studies in which the act of catching a monkey for the purpose of dosing may interfere with immediate response, to chronic toxicity testing in which automatic programming of injections would provide major economic advantages.

\section{References}

Beach, H. D.: Morphine addiction in rats. Canad. J. Psychol. 11, 104-112 (1957). Nichols, J. R., C. P. Headlee, and H. W. Coppock: Drug addiction. I. Addiction by escape training. J. Amer. pharm. Ass. 45, 788-791 (1956).

Seevers, M. H.: Psychopharmacological elements of drug dependence. J. Amer. med. Ass. 206, 1263-1266 (1968).

Thompson, T., and C. R. Schuster: Morphine self-administration, food-reinforced, and avoidance behaviors in thesus monkeys. Psychopharmacologia (Berl.) 5, $87-94$ (1964). 
Weeks,J.R.: Experimental morphine addiction: Method for automatic intravenous injections in unrestrained rats. Science 138, 143-144 (1962).

Wikler, A., W. R. Martin, F. T. Pescor, and C. G. Eades: Factors regulating oral consumption of an opioid (Etonitazine) by morphine-addicted rats. Psychopharmacologia (Berl.) 5, 55-76 (1963).

\author{
G. A. Deneau \\ Drug Abuse Division \\ Southern Research Institute \\ 2000 Ninth Avenue South \\ Birmingham, Alabama 35205, U.S.A.
}

